MPP-2011-21

\title{
Supernova bound on keV-mass sterile neutrinos reexamined
}

\author{
Georg G. Raffelt and Shun Zhou \\ Max-Planck-Institut für Physik (Werner-Heisenberg-Institut), Föhringer Ring 6, D-80805 München, Germany
}

(Dated: 25 February 2011)

\begin{abstract}
Active-sterile neutrino mixing is strongly constrained for $m_{s} \gtrsim 100 \mathrm{keV}$ to avoid excessive energy losses from supernova cores. For smaller $m_{s}$, matter effects suppress the effective mixing angle except for a resonant range of energies where it is enhanced. We study the case of $\nu_{\tau}-\nu_{s}$ mixing where a $\nu_{\tau}-\bar{\nu}_{\tau}$ asymmetry builds up due to the strong excess of $\nu_{s}$ over $\bar{\nu}_{s}$ emission or vice versa, reducing the overall emission rate. In the warm dark matter range $m_{s} \lesssim 10 \mathrm{keV}$ the mixing angle is essentially unconstrained.
\end{abstract}

PACS numbers: 14.60.Pq, 97.60.Bw

\section{INTRODUCTION}

Sterile neutrinos $\nu_{s}$ can be produced in the early universe or in supernova $(\mathrm{SN})$ cores if they mix with one of the active flavors [1, 2]. Even if the mixing angle $\theta$ is very small, repeated collisions of the active component allow for an efficient $\nu_{s}$ production. The SN 1987A neutrino signal duration implies $\sin ^{2} 2 \theta \lesssim 10^{-9}$ to avoid excessive energy losses $3-10$. In detail, this limit depends on whether the dominant mixing is with $\nu_{e}$ or one of the other active flavors. This result assumes $m_{s} \gtrsim 100 \mathrm{keV}$ for matter effects on neutrino propagation to be negligible compared with the vacuum mass.

For smaller masses, the matter effect typically suppresses the effective mixing angle and thus diminishes the limit on $\sin ^{2} 2 \theta$. For $m_{s} \lesssim 1 \mathrm{keV}$ even maximal mixing is allowed. However, no detailed treatment of the SN bound exists for $1 \mathrm{keV} \lesssim m_{s} \lesssim 100 \mathrm{keV}$, where matter and resonance effects are important [4, [6]. On the other hand, this is precisely the mass range where sterile neutrinos could play an interesting warm dark matter role in cosmology [11 13]. While sterile neutrinos can be produced in the early universe by different mechanisms [11, 12], the production by oscillations and collisions once more depends on the active-sterile mixing angle, so one naturally wonders about the SN bound on $\theta$.

In particular, we study the role of feedback of sterile neutrino emission on the emission rate itself. When matter effects are important, the mixing angle is resonantly enhanced in some range of neutrino energies [14 16]. At first the emission of $\bar{\nu}_{s}$ is more efficient than $\nu_{s}$ because in the neutrino sector the mixing angle is suppressed for all energies. As a consequence, the active flavor, being trapped in the SN core, builds up a $\nu$ excess. The opposite could happen for larger mixing angles, when most antineutrinos $\bar{\nu}_{s}$ are trapped rather than freely escape and the emission of $\nu_{s}$ is more efficient than that of $\bar{\nu}_{s}$. To be specific we use $\nu_{\tau}$ as the active flavor because there is no initial $\nu_{\tau}-\bar{\nu}_{\tau}$ asymmetry in a SN core and $m_{\tau}$ is so large that charged $\tau$ leptons never play any role. Our main point is that the depletion of $\nu_{\tau}$ or $\bar{\nu}_{\tau}$ relative to the other always goes in the direction of quenching the initial emission rate, implying that in the $1-100 \mathrm{keV}$-mass range the $\theta$ bounds are indeed suppressed.
Our conclusion differs somewhat from previous discussions where it was suggested that the buildup of a $\nu_{\tau}-\bar{\nu}_{\tau}$ asymmetry goes in the direction of reducing the matter effect and leads to restrictive SN limits on $\theta[10]$. While we agree that the matter effect can be modified in this direction, the positive $\nu_{\tau}-\bar{\nu}_{\tau}$ asymmetry also implies a depletion of source $\bar{\nu}_{\tau}$ to be converted to $\bar{\nu}_{s}$ relative to source $\nu_{\tau}$ to be converted to $\nu_{s}$. It is also possible in some region of the parameter space that the matter effects are enlarged due to a negative $\nu_{\tau}-\bar{\nu}_{\tau}$ asymmetry. In both cases, the compound effect is a reduction, not an enhancement, of the energy loss.

In Sec. II. we briefly review the matter effects on activesterile neutrino mixing in the SN core. The development of a $\nu_{\tau}-\bar{\nu}_{\tau}$ asymmetry is discussed in Sec. III, where we identify a stationary state with equal neutrino and antineutrino emission rates and estimate the time scale to reach it. Sec. IV is devoted to the calculation of the energy-loss rate caused by sterile neutrinos and the SN bound on sterile neutrino masses and mixing angles. Finally, we summarize our conclusions in Sec. V.

\section{MATTER EFFECTS}

The dispersion relation of neutrinos will be modified in matter due to the coherent forward scattering of neutrinos off background particles [16]. This matter effect can be described by an effective potential $V_{\nu_{\alpha}}$ for each kind of active neutrino $\nu_{\alpha}=\nu_{e}, \nu_{\mu}$ and $\nu_{\tau}$. The effective potentials for antineutrinos have the opposite signs, i.e., $V_{\bar{\nu}_{\alpha}}=-V_{\nu_{\alpha}}$. In the case of $\nu_{\tau}-\nu_{s}$ oscillation in matter, the effective Hamiltonian is

$$
H_{\mathrm{eff}}=\left(\begin{array}{cc}
V_{\nu_{\tau}}-\omega c_{2 \theta} & \omega s_{2 \theta} \\
\omega s_{2 \theta} & \omega c_{2 \theta}-V_{\nu_{\tau}}
\end{array}\right)
$$

where $s_{2 \theta} \equiv \sin 2 \theta, c_{2 \theta} \equiv \cos 2 \theta$ with $\theta$ being the vacuum mixing angle and $\omega \equiv \Delta m^{2} / 2 E$ the oscillation frequency in vacuum. As far as the $\mathrm{keV}$-mass sterile neutrinos are concerned, we have $\Delta m^{2} \approx m_{s}^{2}$ with $m_{s}$ being the sterile neutrino mass. In contrast with the flavor conversions of ordinary neutrinos in the SN, there are no collective effects in active-sterile neutrino oscillations. 
In $\mathrm{SN}$ cores, the main ingredients of matter are protons $p$, neutrons $n$, electrons $e$, some muons $\mu$ as well as active neutrinos $\nu_{\alpha}$ and antineutrinos $\bar{\nu}_{\alpha}$. We ignore the possibility of a meson condensate or hyperons. In such a medium, the effective potential for tau neutrinos is

$$
V_{\nu_{\tau}}=\sqrt{2} G_{\mathrm{F}} N_{\mathrm{B}}\left[-\frac{1}{2} Y_{n}+Y_{\nu_{e}}+Y_{\nu_{\mu}}+2 Y_{\nu_{\tau}}\right],
$$

where $G_{\mathrm{F}}$ is the Fermi constant, $N_{\mathrm{B}}$ the baryon number density, and $Y_{X} \equiv\left(N_{X}-N_{\bar{X}}\right) / N_{\mathrm{B}}$ with $N_{X}$ and $N_{\bar{X}}$ being the number densities of particle $X$ and its antiparticle $\bar{X}$. Because of charge neutrality $Y_{p}=Y_{e}+Y_{\mu}$, we have $Y_{n}=1-Y_{e}-Y_{\mu}$. While $\nu_{\alpha}$ and $\bar{\nu}_{\alpha}$ of all flavors can be produced in pairs, electron neutrinos $\nu_{e}$ can also be generated in beta processes such as $e^{-}+p \rightarrow \nu_{e}+n$, and similar for muon neutrinos. The muon mass $m_{\mu}=106 \mathrm{MeV}$ is comparable to the average thermal energy $\langle E\rangle=3 T$ with $T=30 \mathrm{MeV}$, thus a small population of muons is unavoidable. Beta equilibrium $\nu_{\mu}+n \leftrightarrow \mu^{-}+p$ implies the relation among chemical potentials $\mu_{\mu}-\mu_{\nu_{\mu}}=\mu_{n}-\mu_{p} \equiv \hat{\mu}$, where $\hat{\mu}$ typically lies in the range $50-100 \mathrm{MeV}$, depending sensitively on the equation of state. Noting that the initial $\mu$ lepton number is vanishing and taking $\hat{\mu}=50 \mathrm{MeV}$, one finds $\mu_{\mu} \approx 18 \mathrm{MeV}$ and $\mu_{\nu_{\mu}} \approx-32 \mathrm{MeV}$ [17]. On the other hand, the $\nu_{e}$ chemical potential will be much larger due to the electron lepton number trapped during infall.

For simplicity, we consider a SN core just after the bounce and assume the temperature $T=30 \mathrm{MeV}$ and matter density $\rho=3.0 \times 10^{14} \mathrm{~g} \mathrm{~cm}^{-3}$ to be constant. We furthermore take a typical value of electron lepton number fraction $Y_{L}=Y_{e}+Y_{\nu_{e}}=0.37$, which leads to $Y_{e}=0.3$ and $Y_{\nu_{e}}=0.07$ due to beta equilibrium with $\hat{\mu}=50 \mathrm{MeV}$. It is straightforward to verify that $Y_{\mu} / Y_{e}=$ 0.01 and $Y_{\nu_{\mu}} / Y_{\nu_{e}}=-0.05$, so henceforth we simply set $Y_{\mu}=Y_{\nu_{\mu}}=0$. Tau neutrinos initially follow the FermiDirac distribution without chemical potential, but later an asymmetry develops due to $\nu_{s}$ emission. With these simplifications, the effective potential in Eq. (2) is

$$
V_{\nu_{\tau}}=-\frac{G_{\mathrm{F}}}{\sqrt{2}} N_{\mathrm{B}}\left(1-Y_{e}-2 Y_{\nu_{e}}-4 Y_{\nu_{\tau}}\right) .
$$

It is now evident that $V_{\nu_{\tau}}$ is negative for $Y_{\nu_{\tau}}=0$, implying that the Mikheyev-Smirnov-Wolfenstein resonance occurs in the antineutrino sector [14, 15].

Given the effective Hamiltonian in Eq. (1), one immediately obtains the effective mixing angle

$$
\sin ^{2} 2 \theta_{\nu, \bar{\nu}}=\frac{\sin ^{2} 2 \theta}{\sin ^{2} 2 \theta+\left(\cos 2 \theta \pm E / E_{\mathrm{r}}\right)^{2}},
$$

where the upper sign refers to $\nu$ and the lower to $\bar{\nu}$. The resonant energy $E_{\mathrm{r}} \equiv \Delta m^{2} / 2\left|V_{\nu_{\tau}}\right|$ is

$$
E_{\mathrm{r}}=3.25 \mathrm{MeV}\left(\frac{m_{s}}{10 \mathrm{keV}}\right)^{2} \rho_{14}^{-1}\left|Y_{0}-Y_{\nu_{\tau}}\right|^{-1},
$$

where $\rho_{14}$ is the matter density $\rho$ in units of $10^{14} \mathrm{~g} \mathrm{~cm}^{-3}$ and $Y_{0} \equiv\left(1-Y_{e}-2 Y_{\nu_{e}}\right) / 4$. As indicated by Eq. (4), the mixing angle $\theta_{\nu}$ for the whole energy range is always suppressed by matter effects, while $\theta_{\bar{\nu}}$ can be resonantly enhanced for $E \sim E_{\mathrm{r}} \cos 2 \theta$. Note that the "vacuum limit" with $\theta_{\nu} \approx \theta_{\bar{\nu}} \approx \theta$ is reached for large sterile neutrino masses $m_{s} \gg 10 \mathrm{keV}$, while the "medium limit" with reduced mixing angle $\theta_{\nu} \approx \theta_{\bar{\nu}} \approx\left(E_{\mathrm{r}} / E\right) \theta$ is obtained for small masses $m_{s} \ll 10 \mathrm{keV}$. For intermediate masses, we have a resonance in the antineutrino sector.

Sterile neutrinos are produced in the SN core via oscillations and collisions of tau neutrinos. If the effective mixing angles $\theta_{\nu}$ and $\theta_{\bar{\nu}}$ are small enough, $\nu_{s}$ and $\bar{\nu}_{s}$ can escape from the core immediately after production. Since the mixing angle of antineutrinos is always larger than that of neutrinos, the emission rate of antineutrinos exceeds that of neutrinos. Consequently, a $\nu_{\tau}-\bar{\nu}_{\tau}$ asymmetry arises from these different emission rates. As we can observe from Eq. (5), it might turn out that a relatively large $\nu_{\tau}-\bar{\nu}_{\tau}$ asymmetry is achieved, i.e., $Y_{\nu_{\tau}} \rightarrow Y_{0}$, so as to drive the resonant energy $E_{\mathrm{r}}$ to infinity, leading to the "vacuum limit" even for small masses. Put another way, the $\nu_{\tau}-\bar{\nu}_{\tau}$ asymmetry seems to develop in the direction of reducing the matter effects, resulting in restrictive SN limits on the vacuum mixing angle $\theta$ for both large and small sterile neutrino masses. It has been argued [10] that there exists a stationary state with $Y_{\nu_{\tau}}=Y_{0}$, which can be achieved rapidly and thus validates the "vacuum limit" and restrictive bounds on $\theta$.

However, the state with $Y_{\nu_{\tau}}=Y_{0}$ cannot be stationary, because the population of $\nu_{\tau}$ at this moment is larger than that of $\bar{\nu}_{\tau}$, implying that more neutrinos than antineutrinos are ready to be emitted and thus the condition $Y_{\nu_{\tau}}=Y_{0}$ breaks down. Furthermore, it is even possible that the emission rate of $\nu_{s}$ exceeds that of $\bar{\nu}_{s}$, since the effective mixing angle $\theta_{\bar{\nu}}$ can be so large that most of $\bar{\nu}_{s}$ are trapped in the core. Therefore one may obtain a negative asymmetry $Y_{\nu_{\tau}}<0$, driving the system towards the "medium limit." In the following sections, we shall examine how the $\nu_{\tau}-\bar{\nu}_{\tau}$ asymmetry actually develops, and explore its implications on the anomalous energy-loss rate of the SN core and thus the SN bounds on sterile neutrinos.

\section{STATIONARY STATE}

\section{A. Weak-damping limit}

The matter density of SN cores is so high that both neutrino oscillations and frequent collisions with background particles are important. An elegant method to treat neutrino flavor conversions in this case is to implement the matrix of occupation numbers $\left(\rho_{\mathbf{p}}\right)_{i j} \equiv$ $\left\langle b_{j}^{\dagger}(\mathbf{p}) b_{i}(\mathbf{p})\right\rangle$, where $b_{i}(\mathbf{p})$ denotes the annihilation operator for a neutrino of flavor $i$ and momentum $\mathbf{p}$, and to derive the non-Abelian Boltzmann equations of $\rho_{\mathbf{p}}$ 20]. The diagonal elements $\left(\rho_{\mathbf{p}}\right)_{i i}$ are the usual occupation numbers $f_{\mathbf{p}}^{i}$, while the off-diagonal ones encode the phase information. The analogous definition for an- 
tineutrinos is $\left(\bar{\rho}_{\mathbf{p}}\right)_{i j} \equiv\left\langle d_{i}^{\dagger}(\mathbf{p}) d_{j}(\mathbf{p})\right\rangle$ with $d_{i}(\mathbf{p})$ being the annihilation operator for an antineutrino of flavor $i$ and momentum $\mathbf{p}$. In general, the equations of motion for $\rho_{\mathbf{p}}$ and $\bar{\rho}_{\mathbf{p}}$ are complicated by the nonlinear nature of the collision integrals.

For keV-mass sterile neutrinos, the problem can be much simplified by taking the weak-damping limit, which is usually valid in SN cores. To be more explicit, we estimate the neutrino oscillation length in matter

$$
\lambda_{\text {osc }} \lesssim 0.7 \mathrm{~cm}\left(\frac{E}{30 \mathrm{MeV}}\right)\left(\frac{10^{-4}}{\sin 2 \theta}\right)\left(\frac{10 \mathrm{keV}}{m_{s}}\right)^{2}
$$

and the mean free path of tau neutrinos

$$
\lambda_{\mathrm{mfp}}=\frac{1}{N_{\mathrm{B}} \sigma_{\nu N}} \approx 1.1 \times 10^{3} \mathrm{~cm}\left(\frac{30 \mathrm{MeV}}{E}\right)^{2} \rho_{14}^{-1},
$$

where $\sigma_{\nu N} \sim G_{\mathrm{F}}^{2} E^{2} / \pi$ is the cross section of neutrinonucleon scattering via the neutral-current interaction. The weak-damping limit is $\lambda_{\text {osc }} \ll \lambda_{\text {mfp }}$, meaning that the active-sterile neutrino oscillations take place many times before a subsequent collision of active neutrinos with the nucleons. It deserves mention that the weakdamping limit is violated for smaller masses $m_{s} \ll 1 \mathrm{keV}$, however, the effective neutrino mixing angle in this case is highly suppressed by matter effects and thus the energyloss rate is negligibly small. We shall always assume the weak-damping limit in the mass range of our interest.

\section{B. Emission rates}

In the weak-damping limit, $\rho_{\mathbf{p}}$ is averaged over many cycles of oscillations and then can be parametrized by the neutrino occupation numbers $f_{E}^{\tau}$ and $f_{E}^{s}$ with the neutrino energy $E=|\mathbf{p}|$ and similar for antineutrinos [4]. Note that we consider a homogenous and isotropic ensemble of active neutrinos and antineutrinos, which are trapped in the SN core and stay in thermal equilibrium with ambient matter, so the momentum direction is irrelevant. Assuming that sterile neutrinos freely escape, we obtain the evolution equation of the $\nu_{\tau}$ density

$$
\dot{N}_{\nu_{\tau}}=-\frac{1}{4} \sum_{a} \int \frac{E^{2} \mathrm{~d} E}{2 \pi^{2}} s_{2 \theta_{\nu}}^{2} \int \frac{E^{\prime 2} \mathrm{~d} E^{\prime}}{2 \pi^{2}} W_{E^{\prime} E}^{a} f_{E^{\prime}}^{\tau},
$$

where $a$ denotes the target particle, and $W_{E^{\prime} E}^{a}$ the transition probability for $\nu_{\tau}\left(E^{\prime}\right)+a \rightarrow \nu_{\tau}(E)+a$ via the neutral-current interaction. In a similar way, we can derive the evolution equation of the $\bar{\nu}_{\tau}$ number density, involving the mixing angle $\theta_{\bar{\nu}}$, the occupation number $f_{E}^{\bar{\tau}}$ and the transition probability $\bar{W}_{E^{\prime} E}^{a}$. However, only the neutrino energies ensuring $\theta_{\nu}, \theta_{\bar{\nu}} \lesssim \theta_{c}$ are taken under the integration, where the critical mixing angle $\theta_{c} \sim 10^{-2}$ can be estimated by requiring the mean free path of sterile neutrinos to equal the core radius $R \sim 10 \mathrm{~km}$. On the other hand, the transition probabilities $W_{E^{\prime} E}^{a}$ and
$\bar{W}_{E^{\prime} E}^{a}$ of neutrino-nucleon scattering dominate over those of neutrino-electron scattering if the sterile neutrino mass is lying in the range of $1 \mathrm{keV} \lesssim m_{s} \lesssim 100 \mathrm{keV}$. Hence we consider only the neutral-current $\nu$ - $N$ scattering in the leading-order approximation.

\section{Degeneracy parameter}

In order to describe the $\nu_{\tau}-\bar{\nu}_{\tau}$ asymmetry, we assume the occupation number $f_{E}^{\tau}(t)=[\exp (E / T-\eta(t))+1]^{-1}$ for $\nu_{\tau}$ and $f_{E}^{\bar{\tau}}(t)=[\exp (E / T+\eta(t))+1]^{-1}$ for $\bar{\nu}_{\tau}$, where $T$ is the temperature of the SN core and $\eta(t)$ is the degeneracy parameter. In the absence of active-sterile neutrino mixing, the spectra of $\nu_{\tau}$ and $\bar{\nu}_{\tau}$ just follow the FermiDirac distribution with a vanishing chemical potential, namely $\eta=0$ at $t=0$. Substituting the occupation numbers into Eq. (8), taking account of the neutral-current $\nu_{\tau^{-}} N$ scattering and subtracting the corresponding equation for antineutrinos, one arrives at

$$
\frac{\mathrm{d} \eta(t)}{\mathrm{d} t}=\frac{N_{\mathrm{B}} G_{\mathrm{F}}^{2} s_{2 \theta}^{2} T^{2}}{4 \pi}\left[\mathscr{F}_{\bar{\nu}}(\eta)-\mathscr{F}_{\nu}(\eta)\right] \mathscr{G}^{-1}(\eta),
$$

where the relevant functions are defined as follows

$$
\begin{aligned}
& \mathscr{F}_{\bar{\nu}}(\eta)=\int_{0}^{\infty} \frac{x^{4}}{e^{x+\eta}+1} \frac{1-\mathscr{B}\left(x, x_{\mathrm{r}} \epsilon^{-}, x_{\mathrm{r}} \epsilon^{+}\right)}{s_{2 \theta}^{2}+\left(c_{2 \theta}-x / x_{\mathrm{r}}\right)^{2}} \mathrm{~d} x, \\
& \mathscr{F}_{\nu}(\eta)=\int_{0}^{\infty} \frac{x^{4}}{e^{x-\eta}+1} \frac{\Theta\left(x+x_{\mathrm{r}} \epsilon^{-}\right)}{s_{2 \theta}^{2}+\left(c_{2 \theta}+x / x_{\mathrm{r}}\right)^{2}} \mathrm{~d} x,
\end{aligned}
$$

and $\mathscr{G}(\eta) \equiv \mathrm{d}\left[F_{2}(\eta)-F_{2}(-\eta)\right] / \mathrm{d} \eta$ with $F_{2}(\eta)$ being the Fermi-Dirac integral of order two. In addition, we have introduced $x_{\mathrm{r}} \equiv E_{\mathrm{r}} / T, \epsilon^{ \pm} \equiv s_{2\left(\theta_{c} \pm \theta\right)} / s_{2 \theta_{c}}$, and the box function $\mathscr{B}(x, a, b) \equiv \Theta(x-a)-\Theta(x-b)$, which equals one for $x \in[a, b]$ and vanishes otherwise. Here $\Theta(x)$ denotes the unit step function, i.e., $\Theta(x)=0$ for $x<0$ and $\Theta(x)=1$ for $x \geq 0$. Note that Eq. (10) has been cast into a compact form so as to include both $\theta \leq \theta_{c}$ and $\theta>\theta_{c}$ cases.

Taking typical values of the matter density $\rho_{14}=3.0$ and the core temperature $T=30 \mathrm{MeV}$, we can rewrite Eq. (9) as $\dot{\eta}(t)=\tau_{0}^{-1} \mathscr{H}(\eta)$ with $\tau_{0}=1 \mathrm{~s}\left(10^{-8} / s_{2 \theta}^{2}\right)$ and $\mathscr{H}(\eta)=\left[\mathscr{F}_{\bar{\nu}}(\eta)-\mathscr{F}_{\nu}(\eta)\right] \mathscr{G}^{-1}(\eta)$. The time evolution of the degeneracy parameter $\eta(t)$ depends crucially on the initial difference between neutrino and antineutrino emission rates, i.e., $\tau_{0}^{-1} \mathscr{H}(0)$, as well as the evolution of $\mathscr{H}(\eta)$ with respect to $\eta$. In Fig. 1, we show the initial rate $\dot{\eta}(0)=\tau_{0}^{-1} \mathscr{H}(0)$ in the $\left(\sin ^{2} 2 \theta, m_{s}\right)$ plane, where the blank region with "+" denotes a strong excess of $\bar{\nu}_{s}$ over $\nu_{s}$ emission while that with "-" represents the opposite case. The cyan regions on the left-hand and right-hand side indicate $\dot{\eta}(0)>0$ and $\dot{\eta}(0)<0$, respectively, but the magnitude of $|\dot{\eta}(0)|$ is extremely small for both cases. The reason for the former case is just that the effective mixing angle is too small, and for the latter case is that most of $\nu_{s}$ and $\bar{\nu}_{s}$ are trapped.

If the initial emission rate of $\bar{\nu}_{s}$ is larger than that of $\nu_{s}$, i.e., $\dot{\eta}(0)>0$, the degeneracy parameter increases from 


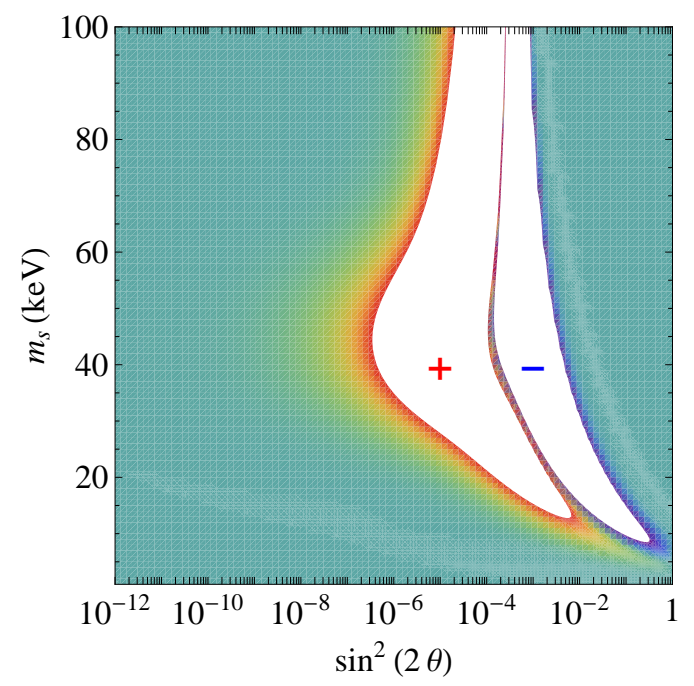

FIG. 1: Density plot of the initial rate $\dot{\eta}(0)=\tau_{0}^{-1} \mathscr{H}(0)$, where the blank region with "+" stands for a strong excess of $\bar{\nu}_{s}$ over $\nu_{s}$ emission while that with "-" is the other way round. The narrow strip indicates a rapid and continuous transition between these two regions.

zero to a positive stable value $\eta^{*}$, at which the emission rates of $\nu_{s}$ and $\bar{\nu}_{s}$ are equal, namely $\mathscr{F}_{\bar{\nu}}\left(\eta^{*}\right)=\mathscr{F}_{\nu}\left(\eta^{*}\right)$ or equivalently $\mathscr{H}\left(\eta^{*}\right)=0$. In this case, we are finally left with a positive $\nu_{\tau}-\bar{\nu}_{\tau}$ asymmetry when such a stationary state is reached. If the initial emission rate of $\nu_{s}$ exceeds that of $\bar{\nu}_{s}$, i.e., $\dot{\eta}(0)<0$, the degeneracy parameter decreases from zero to a negative stable value $\eta^{*}$. At this moment, we have equal neutrino and antineutrino emission rates as well, but a negative $\nu_{\tau}-\bar{\nu}_{\tau}$ asymmetry.

The important point here is feedback of the established $\nu_{\tau}-\bar{\nu}_{\tau}$ asymmetry or a finite degeneracy parameter. In the case of $\dot{\eta}(0)>0$, a positive $\eta$ suppresses the $\bar{\nu}_{\tau}$ population and shifts the resonant energy to a larger value. The combined result is just to reduce the $\bar{\nu}_{s}$ emission rate. Meanwhile, the population of $\nu_{\tau}$ is accordingly increased and the mixing angle $\theta_{\nu}$ becomes less suppressed, enhancing the $\nu_{s}$ emission rate. Similar arguments apply to the case of $\dot{\eta}(0)<0$. It is also possible that $\dot{\eta}(0)=0$, which lies in the narrow strip in Fig. 1, the system remains in its initial state with a vanishing $\nu_{\tau}-\bar{\nu}_{\tau}$ asymmetry. Hence we have no feedback effect in this special case.

In Fig. 2, the degeneracy parameter $\eta^{*}$ of the stationary state has been solved from $\mathscr{F}_{\bar{\nu}}(\eta)=\mathscr{F}_{\nu}(\eta)$, no matter whether such a stationary state can be reached. The large values of $\eta^{*}$ appear in the region where the initial emission rate of $\nu_{s}$ is significantly different from that of $\bar{\nu}_{s}$. This condition can be satisfied for (1) small mixing angles and intermediate masses, when the mixing angle $\theta_{\bar{\nu}}$ is resonantly enhanced so that $\mathscr{F}_{\bar{\nu}}(0) \gg \mathscr{F}_{\nu}(0)$; (2) large mixing angles and large masses, when more $\bar{\nu}_{s}$ than $\nu_{s}$ are trapped in the core such that $\mathscr{F}_{\bar{\nu}}(0) \ll$ $\mathscr{F}_{\nu}(0)$. However, it is obvious that the $\nu_{s}$ and $\bar{\nu}_{s}$ emission rates in both cases are extremely small.

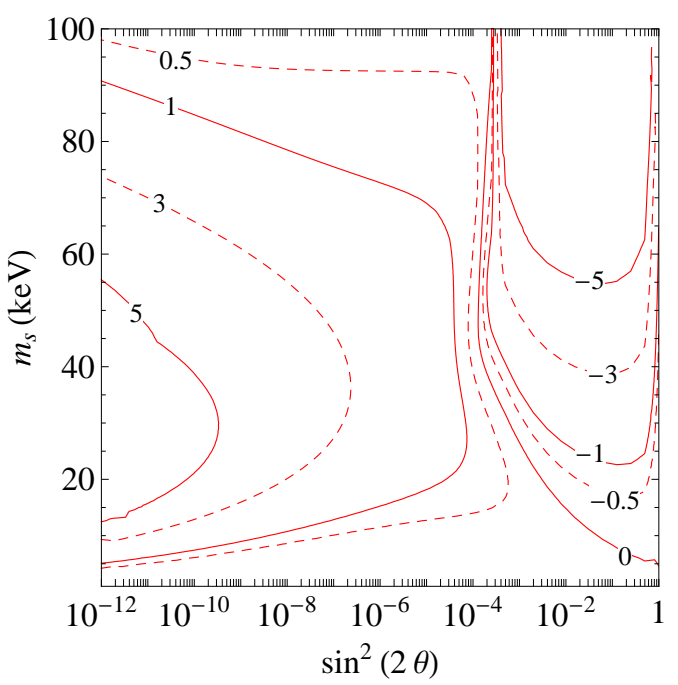

FIG. 2: Contour plot of the asymptotic degeneracy parameter $\eta^{*}$, which is determined by $\mathscr{F}_{\bar{\nu}}\left(\eta^{*}\right)=\mathscr{F}_{\nu}\left(\eta^{*}\right)$, implying equal neutrino and antineutrino emission rates.

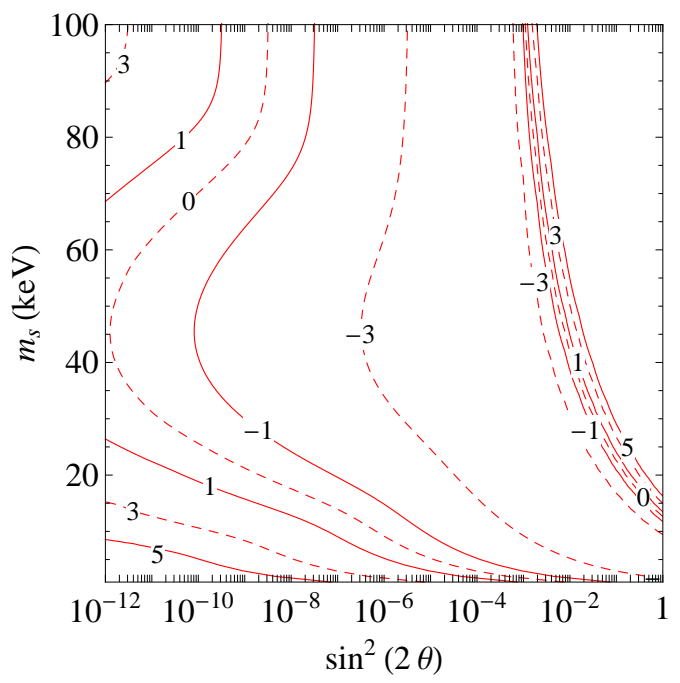

FIG. 3: Contour plot of the estimated timescale $\tau=$ $\eta^{*} /\left[\tau_{0}^{-1} \mathscr{H}(0)\right]$ to reach the stationary state, where the contours are labeled by the $\operatorname{logarithmic}$ values $\log (\tau / 1 \mathrm{~s})$.

The timescale for the system to achieve the stationary state can be determined by numerically solving Eq. (9). For a rough estimate, we take $\dot{\eta}(t) \sim \dot{\eta}(0)=\tau_{0}^{-1} \mathscr{H}(0)$ and then obtain the timescale $\tau=\eta^{*} /\left[\tau_{0}^{-1} \mathscr{H}(0)\right]$. In Fig. 3, we show the estimated timescale $\tau$ in the $\left(\sin ^{2} 2 \theta, m_{s}\right)$ plane. In the mass range $1 \mathrm{keV} \lesssim m_{s} \lesssim$ $10 \mathrm{keV}$, where sterile neutrinos can be warm dark matter, the timescale is larger than the neutrino diffusion time $\tau_{\mathrm{d}}=1 \mathrm{~s}$ for small mixing angles $\sin ^{2} 2 \theta \lesssim 10^{-6}$. For larger mixing angles, the stationary state can be achieved within a fraction of a second, but $\eta^{*}$ in this case is quite small as shown in Fig. 2. Hence we expect that the feedback effects are negligible. In this con- 


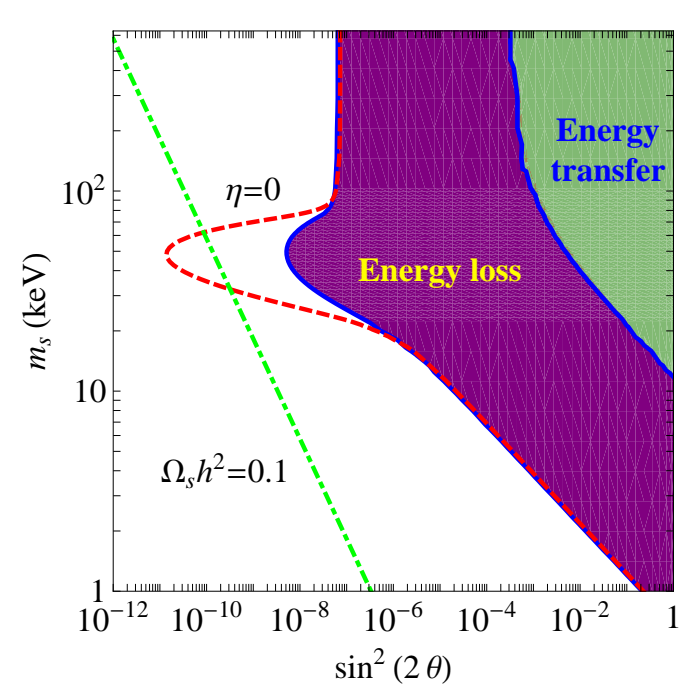

FIG. 4: Supernova bound on sterile neutrino masses $m_{s}$ and mixing angles $\theta$, where the purple region is excluded by the energy-loss argument while the green one by the energytransfer argument. The excluded region will be extended to the dashed (red) line if the build-up of degeneracy parameter is ignored, i.e., $\eta(t)=0$. The dot-dashed (green) line represents the sterile neutrinos as dark matter with the correct relic abundance $\Omega_{s} h^{2}=0.1$.

nection, the most interesting parameter space should be $20 \mathrm{keV} \lesssim m_{s} \lesssim 80 \mathrm{keV}$ and $10^{-9} \lesssim \sin ^{2} 2 \theta \lesssim 10^{-4}$, where both sizable $\eta^{*}$ and $\tau<\tau_{\mathrm{d}}$ are expected. Since $|\mathscr{H}(\eta)|$ decreases from the initial value $|\mathscr{H}(0)|$ to zero, as the absolute value of the degeneracy parameter $|\eta(t)|$ increases from zero to $\left|\eta^{*}\right|$, the relaxation time may be underestimated. However, this rough estimate has already shown the main features of the relaxation timescale.

\section{SUPERNOVA BOUNDS}

The emission of sterile neutrinos $\nu_{s}$ and $\bar{\nu}_{s}$ may cause rapid energy losses from the SN cores, which can significantly shorten the duration of neutrino signals 21]. In order to avoid conflict with the observation of SN 1987A neutrinos, we require the energy-loss rate per unit mass to be smaller than $1.0 \times 10^{19} \mathrm{erg} \mathrm{g}^{-1} \mathrm{~s}^{-1}$, which can be translated into the volume energy-loss rate $\mathscr{E}<3.0 \times 10^{33} \mathrm{erg} \mathrm{cm}^{-3} \mathrm{~s}^{-1}$ for $\rho_{14}=3.0$. Given the emission rate of neutrinos in Eq. (8) and the counterpart for antineutrinos, the energy-loss rate is

$$
\mathscr{E}(t)=\frac{N_{\mathrm{B}} G_{\mathrm{F}}^{2} s_{2 \theta}^{2} T^{6}}{8 \pi^{3}}\left[\mathscr{R}_{\bar{\nu}}(\eta)+\mathscr{R}_{\nu}(\eta)\right]
$$

with

$$
\begin{aligned}
& \mathscr{R}_{\bar{\nu}}(\eta)=\int_{0}^{\infty} \frac{x^{5}}{e^{x+\eta}+1} \frac{1-\mathscr{B}\left(x, x_{\mathrm{r}} \epsilon^{-}, x_{\mathrm{r}} \epsilon^{+}\right)}{s_{2 \theta}^{2}+\left(c_{2 \theta}-x / x_{\mathrm{r}}\right)^{2}} \mathrm{~d} x \\
& \mathscr{R}_{\nu}(\eta)=\int_{0}^{\infty} \frac{x^{5}}{e^{x-\eta}+1} \frac{\Theta\left(x+x_{\mathrm{r}} \epsilon^{-}\right)}{s_{2 \theta}^{2}+\left(c_{2 \theta}+x / x_{\mathrm{r}}\right)^{2}} \mathrm{~d} x
\end{aligned}
$$

where the definitions of relevant parameters are given below Eq. (10). The energy-loss rate $\mathscr{E}(t)$ depends on time through the degeneracy parameter $\eta(t)$, for which the time evolution has been discussed in last section.

To constrain the sterile neutrino mass and mixing angle, we evaluate the emission rate

$$
\langle\mathscr{E}\rangle=\tau_{\mathrm{d}}^{-1} \int_{0}^{\tau_{\mathrm{d}}} \mathscr{E}(t) \mathrm{d} t
$$

averaged over the neutrino diffusion timescale $\tau_{\mathrm{d}}=1 \mathrm{~s}$. Beyond the diffusion timescale, one may expect that all active neutrinos have already diffused out of the core and thus the emission of sterile neutrinos is physically meaningless. Our strategy is to follow the time evolution of $\eta(t)$ for each point in the $\left(\sin ^{2} 2 \theta, m_{s}\right)$ parameter space, and then calculate the averaged energy-loss rate in Eq. (13). We show in Fig. 4 the contour plot of the averaged energy-loss rate $\langle\mathscr{E}\rangle$ in the $\left(\sin ^{2} 2 \theta, m_{s}\right)$ plane, where the purple region corresponds to $\langle\mathscr{E}\rangle\rangle$ $3.0 \times 10^{33} \mathrm{erg} \mathrm{cm}^{-3} \mathrm{~s}^{-1}$ and is thus excluded.

Some comments are in order. First, the initial energyloss rate with $\eta=0$ is shown in Fig. 4 for comparison. Except for $20 \mathrm{keV} \lesssim m_{s} \lesssim 100 \mathrm{keV}$ and small mixing angles, the averaged energy-loss rate $\langle\mathscr{E}\rangle$ cannot be distinguished from the initial one $\mathscr{E}(0)$. The reason is that either the stationary state has not been reached within $1 \mathrm{~s}$, or the asymptotic value $\eta^{*}$ for the stationary state is quite small. In the mass range $20 \mathrm{keV}<m_{s}<100 \mathrm{keV}$, the buildup of a $\nu_{\tau}-\bar{\nu}_{\tau}$ asymmetry is efficient and strongly reduces the energy-loss rate.

Second, keV-mass sterile neutrinos can be produced in the early universe and contribute as dark matter to the total energy density. In a nonresonant production scheme without large primordial lepton asymmetries, the relic sterile neutrino abundance can be estimated as [10, 13]

$$
\Omega_{s} h^{2} \approx 0.3\left(\frac{\sin ^{2} 2 \theta}{10^{-10}}\right)\left(\frac{m_{s}}{100 \mathrm{keV}}\right)^{2} .
$$

The correct dark matter abundance $\Omega_{s} h^{2}=0.1$ is shown in Fig. 4, where one can see that the masses around $m_{s}=50 \mathrm{keV}$ have been excluded by the SN bound if the degeneracy parameter is assumed to be vanishing. In a realistic situation, this region is retrieved because the energy-loss rate is reduced as the $\nu_{\tau}-\bar{\nu}_{\tau}$ asymmetry builds up. However, the warm dark matter range $1 \mathrm{keV} \lesssim m_{s} \lesssim 10 \mathrm{keV}$ is essentially unconstrained. For sterile neutrinos of masses below $1 \mathrm{keV}$, even maximal mixing is allowed, because the total energy-loss rate is highly suppressed by matter effects.

Finally, our discussion was based on the energy-loss argument, ignoring the sterile neutrinos trapped in the $\mathrm{SN}$ core. For this reason, the top-right green region in Fig. 4, where both the sterile neutrino mass and vacuum mixing angle are large, was not excluded. However, the mean free path of these trapped sterile neutrinos is much larger than that of $\nu_{\tau}$, so the energy transfer in the SN core will be more efficient. The energy in a $\mathrm{SN}$ core is 
carried by those trapped particles with the largest mean free path, a role played here by the sterile states. Once more the neutrino burst duration is shortened too much if the sterile mfp is larger than a few times the one for $\nu_{\tau}$ [7]. Therefore, the large mixing angle region is actually excluded in the spirit of the energy-transfer argument.

\section{CONCLUSIONS}

Since keV-mass sterile neutrinos are a promising candidate for warm dark matter, we have revisited the supernova bound on the sterile neutrino masses and mixing angles by studying the case of $\nu_{\tau}-\nu_{s}$ mixing in the SN core and requiring no excessive energy losses induced by sterile neutrinos. It turns out that the warm dark matter range is essentially unconstrained, while sterile neutrinos of masses around $50 \mathrm{keV}$ receive the most stringent constraint, i.e., $\sin ^{2} 2 \theta \lesssim 4.0 \times 10^{-9}$. For even larger masses $m_{s} \gtrsim 100 \mathrm{keV}$, the SN limit on the mixing angles is $\sin ^{2} 2 \theta \lesssim 5.0 \times 10^{-8}$, which is about one order of magnitude weaker than that for $m_{s} \sim 50 \mathrm{keV}$. It is the matter effects that render these constraints quite different.

We have identified a mass range $20 \mathrm{keV} \lesssim m_{s} \lesssim$ $100 \mathrm{keV}$ where a sizable $\nu_{\tau}-\bar{\nu}_{\tau}$ asymmetry can be established due to the strong excess of $\bar{\nu}_{s}$ over $\nu_{s}$ emission or vice versa. The build-up of this asymmetry feeds back on the emission rates, leading to a stationary state where the neutrino and antineutrino emissions become equal. For proper mixing angles, such a stationary state can be achieved within the neutrino diffusion timescale $\tau_{\mathrm{d}}=1 \mathrm{~s}$. As a consequence, the energy-loss rate will be significantly reduced, and thus the bounds are relaxed.

As for the $\nu_{\mu}-\nu_{s}$-mixing case, our discussions about the feedback effects are essentially applicable. However, the charged-current interactions of $\nu_{\mu}$ and $\bar{\nu}_{\mu}$ should be taken into account, and the change of $\nu_{\mu}-\bar{\nu}_{\mu}$ asymmetry will be redistributed between muon neutrinos and charged muons. The $\nu_{e}-\nu_{s}$ mixing in SN cores is more involved because of the large trapped electron number and high $\nu_{e}$ degeneracy. Besides energy loss, deleptonization by sterile neutrino emission is an effect to be taken into account. This case requires a dedicated investigation.

We have performed a "single zone" analysis by assuming a homogenous and isotropic SN core with constant matter density and temperature. This treatment should capture the dominant feedback effect. However, the local variation of these quantities may modify the final results, for example smearing out the resonance in the energy-loss rate. Such a refinement is also left for future works.

\section{Acknowledgements}

This work was partly supported by the Deutsche Forschungsgemeinschaft under Grants No. TR-27 and No. EXC-153 and by the Alexander von Humboldt Foundation.
[1] A. Kusenko, Phys. Rept. 481, 1 (2009) arXiv:0906.2968.

[2] A. Boyarsky, O. Ruchayskiy and M. Shaposhnikov, Ann. Rev. Nucl. Part. Sci. 59, 191 (2009) arXiv:0901.0011.

[3] K. Kainulainen, J. Maalampi and J. T. Peltoniemi, Nucl. Phys. B 358, 435 (1991).

[4] G. Raffelt and G. Sigl, Astropart. Phys. 1, 165 (1993) astro-ph/9209005.

[5] J. T. Peltoniemi, Astron. Astrophys. 254, 121 (1992).

[6] X. Shi and G. Sigl, Phys. Lett. B 323, 360 (1994), (E) ibid. B 324, 516 (1994) hep-ph/9312247.

[7] A. D. Dolgov, S. H. Hansen, G. Raffelt and D. V. Semikoz, Nucl. Phys. B 590, 562 (2000) hep-ph/0008138.

[8] A. D. Dolgov, S. H. Hansen, G. Raffelt and D. V. Semikoz, Nucl. Phys. B 580, 331 (2000) arXiv:hep-ph/0002223.

[9] A. D. Dolgov and S. H. Hansen, Astropart. Phys. 16, 339 (2002) hep-ph/0009083.

[10] K. Abazajian, G. M. Fuller and M. Patel, Phys. Rev. D 64, 023501 (2001) astro-ph/0101524.

[11] S. Dodelson and L. M. Widrow, Phys. Rev. Lett. 72, 17
(1994) hep-ph/9303287.

[12] X. D. Shi and G. M. Fuller, Phys. Rev. Lett. 82, 2832 (1999) astro-ph/9810076.

[13] T. Asaka, M. Laine and M. Shaposhnikov, JHEP 0701, 091 (2007) arXiv:hep-ph/0612182.

[14] L. Wolfenstein, Phys. Rev. D 17, 2369 (1978).

[15] S. P. Mikheyev and A. Yu. Smirnov, Sov. J. Nucl. Phys. 42, 913 (1985) [Yad. Fiz. 42, 1441 (1985)].

[16] T. K. Kuo and J. T. Pantaleone, Rev. Mod. Phys. 61, 937 (1989).

[17] S. Hannestad, H. T. Janka, G. G. Raffelt and G. Sigl, Phys. Rev. D 62, 093021 (2000) astro-ph/9912242.

[18] L. Stodolsky, Phys. Rev. D 36, 2273 (1987).

[19] G. Raffelt, G. Sigl and L. Stodolsky, Phys. Rev. Lett. 70, 2363 (1993), (E) ibid. 98, 069902 (2007)] hep-ph/9209276.

[20] G. Sigl and G. Raffelt, Nucl. Phys. B 406, 423 (1993).

[21] G. G. Raffelt, Phys. Rept. 198, 1 (1990). 